\title{
Design of a mutation-integrated trimeric RBD with broad protection against SARS-CoV-2
}

\author{
Yu Liang ${ }^{1,2}$, Jing Zhang ${ }^{1,2}$, Run Yu Yuan ${ }^{3}$, Mei Yu Wang ${ }^{4}$, Peng He ${ }^{4}$, Ji Guo Su ${ }^{1,2}$, Zi Bo Han ${ }^{1,2}$, Yu Qin Jin ${ }^{1,2}$, \\ Jun Wei Hou ${ }^{1,2}$, Hao Zhang ${ }^{1,2}$, Xue Feng Zhang ${ }^{1,2}$, Shuai Shao ${ }^{1,2}$, Ya Nan Hou ${ }^{1,2}$, Zhao Ming Liu ${ }^{1,2}$, Li Fang Du ${ }^{1,2}$,

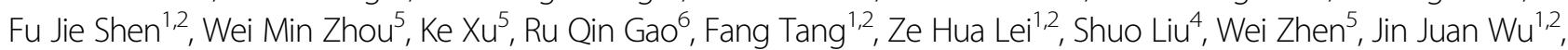 \\ Xiang Zheng ${ }^{1,2}$, Ning Liu ${ }^{1,2}$, Shi Chen ${ }^{1,2}$, Zhi Jing Ma ${ }^{1,2}$, Fan Zheng ${ }^{1,2}$, Si Yu Ren ${ }^{1,2}$, Zhong Yu Hu ${ }^{4 凶}$,
}

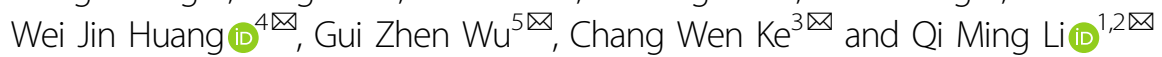

\begin{abstract}
The continuous emergence of SARS-CoV-2 variants highlights the need of developing vaccines with broad protection. Here, according to the immune-escape capability and evolutionary convergence, the representative SARS-CoV-2 strains carrying the hotspot mutations were selected. Then, guided by structural and computational analyses, we present a mutation-integrated trimeric form of spike receptor-binding domain (mutl-tri-RBD) as a broadly protective vaccine candidate, which combined heterologous RBDs from different representative strains into a hybrid immunogen and integrated immune-escape hotspots into a single antigen. When compared with a homo-tri-RBD vaccine candidate in the stage of phase II trial, of which all three RBDs are derived from the SARS-CoV-2 prototype strain, mutltri-RBD induced significantly higher neutralizing antibody titers against the Delta and Beta variants, and maintained a similar immune response against the prototype strain. Pseudo-virus neutralization assay demonstrated that mutl-triRBD also induced broadly strong neutralizing activities against all tested 23 SARS-CoV-2 variants. The in vivo protective capability of mutl-tri-RBD was further validated in hACE2-transgenic mice challenged by the live virus, and the results showed that mutl-tri-RBD provided potent protection not only against the SARS-CoV-2 prototype strain but also against the Delta and Beta variants.
\end{abstract}

\section{Introduction}

Severe acute respiratory syndrome coronavirus 2 (SARS-CoV-2) has spread rapidly around the world since the end of 2019 and resulted in a pandemic of this new coronavirus infections ${ }^{1,2}$, which has imposed a big threat to public health and a heavy burden on the global economy. To date, significant achievement has been made in the coronavirus disease 2019 (COVID-19) vaccine development and several vaccines are authorized by World

Correspondence: Zhong Yu Hu (huzhy@nifdc.org.cn) or Wei

Jin Huang (huangweijin@nifdc.org.cn) or Gui Zhen Wu (wugz@ivdc.chinacdc.cn) or Chang Wen Ke (kecw1965@aliyun.com) or Qi Ming Li (liqiming189@163.com) ${ }^{1}$ The Sixth Laboratory, National Vaccine and Serum Institute (NVSI), Beijing, China ${ }^{2}$ National Engineering Center for New Vaccine Research, Beijing, China

Full list of author information is available at the end of the article

These authors contributed equally: Yu Liang, Jing Zhang, Run Yu Yuan, Mei Yu Wang, Peng He
Health Organization (WHO) for emergency use $\mathrm{us}^{3-5}$. However, new variants are continuously emerging due to the fast evolution of the virus. As of October 2021, four SARS-CoV-2 strains, including Alpha (B.1.1.7), Beta (B.1.351), Gamma (P.1), and Delta (B.1.617.2), are classified as variants of concern by WHO. More lines of evidence have demonstrated the increased transmissibility and virulence of these viriants ${ }^{6-8}$. These mutations may also potentially lead to immune escape from the host defense system ${ }^{9}$. The Beta and Gamma variants have been proved to be able to evade the neutralizing antibodies elicited by natural infections and vaccinations ${ }^{10}$. The ChAdOx1 $\mathrm{nCoV}-19$ vaccine has been proved in clinical trials to be unprotective against the mild-to-moderate COVID-19 caused by the Beta variant ${ }^{11}$. The emergence of SARS-CoV-2 variants within the lineage B.1.617, 
including Kappa (B.1.617.1), Delta (B.1.617.2), and B.1.617.3, has led to a new wave of infections, and several preliminary studies suggested that the B.1.617 variants were less sensitive to the sera from vaccinated individuals and resistant to some monoclonal antibodies ${ }^{12,13}$. Exhaustive analysis of the available SARS-CoV-2 sequences has revealed that several immune-resistant mutations in the circulating variants were also evolutionarily convergent hotspots $^{14}$, indicating the possible recurrence of these mutations individually or combinedly in the future. The possible immune escape of the emerging SARS-CoV-2 variants has raised concerns on the efficacy of existing vaccines and raised urgent needs of developing vaccines with broad-spectrum protection against variants evolved.

The conventional method for the development of a multi-protective vaccine is to produce multivalent vaccines through a mixture of strain-specific monovalent vaccines together. But this strategy is time-consuming and cost-expensive. Another effective method to develop multivalent vaccine is the construction of chimeric vaccine via integrating multiple circulating variants into a single antigen by structure- and computation-based rational design. The commonly used strategy in chimeric protein design is the replacement of structurally homologous modular domains without changing the overall structure of the antigenic protein, e.g., a chimeric Sarbecovirus spike protein constructed by Martinez et al. ${ }^{15}$. However, for the highly variable virus, another more promising strategy for broadly protective vaccine design is to integrate rather than substitute immunodominant domains into a single immunogen.

The spike (S) glycoproteins on SARS-CoV-2 surface play a key role in recognition of the host cell receptor, i.e., human angiotensin-converting enzyme 2 (hACE2), to mediate virus entry into the cell. S protein is naturally self-assembled into a homotrimer anchored onto the viral membrane. Each monomer of $\mathrm{S}$ protein is composed of two subunits, $\mathrm{S} 1$ and $\mathrm{S} 2^{16,17}$. The receptor-binding domain (RBD) of $\mathrm{S} 1$ subunit protrudes from the viral surface and directly interacts with the receptor hACE2. Immunological studies showed that S1 RBD contains multiple neutralizing epitopes, which are immunodominant in eliciting neutralizing antibody responses against SARS-CoV-2 infection ${ }^{18-20}$. In addition, S1 RBD-based vaccine candidates have been shown to elicit neutralizing antibody responses in vitro against both the pseudo and live SARS-CoV-2, and induce protective immunity in vivo in non-human primates ${ }^{21,22}$. However, the RBD monomer has limitations in immunogenicity for vaccine development due to its small molecular size ${ }^{23,24}$. To improve the immunogenicity of RBD, polymerization would be a potentially effective strategy by increasing its molecular size to develop the immunogen with multiple copies of antigenic determinants. Given that native $\mathrm{S}$ protein is selfassembled into a trimer and S1 RBDs naturally exist in a homo-trimeric form, it is reasonably speculated that a trimeric form of RBD resembling its native conformational arrangements could achieve better immunogenic properties. Structural analysis of $\mathrm{S}$ trimer indicates that the $\mathrm{N}$ - and C-terminus of RBD are close to each other and there exists a long loop with high flexibility at both termini, which potentially allow the formation of RBD trimer (tri-RBD) through end-to-end connection to mimic the RBD arrangements in the natural S protein trimer. Furthermore, the tri-RBD that accommodates three RBDs in one molecule enables us to construct a multivalent vaccine candidate, in which three RBDs are individually derived from different circulating SARS-CoV-2 variants and co-assembled into one hybrid trimer.

In the present study, according to the immune-escape capability and evolutionary convergence, the representative SARS-CoV-2 circulating strains that harbor the hotspot mutations were selected. Then, guided by structural and computational analyses, we designed a mutation-integrated trimeric form of $\mathrm{RBD}$, named mutI-tri-RBD, as a vaccine candidate with broadly protective capability, in which the RBDs from the representative SARS-CoV-2 strains were connected end-to-end and co-assembled to possibly mimic the native trimeric arrangements in the natural $S$ protein trimer. It is worth pointing out that a homo-tri-RBD vaccine candidate developed based on SARS-CoV-2 prototype strain by our group is currently in phase II clinical trial (NCT04869592). Preliminary results of the trial demonstrated that homo-tri-RBD is safe and well tolerated. The neutralizing antibody geometric mean titers (GMTs) after three vaccine doses in the participants were about 3-5-fold higher than those of human convalescent sera (data not yet published). In the present study, the homo-tri-RBD was used as a comparative control. Then, the biochemical characterizations and immunological properties of the recombinant mutI-tri-RBD were evaluated comprehensively and compared with those of homo-tri-RBD, which support the mutI-tri-RBD as a promising immunogen for further clinical developments.

\section{Results}

\section{Structure and computation-guided construction of the mutl-tri-RBD as an immunogen}

A hybrid trimeric RBD named mutI-tri-RBD was constructed by incorporating multiple immunodominant RBDs from different circulating variants into a single immunogenic molecule. The rationale for the design of mutI-tri-RBD is as follows: (1) In the native $S$ protein, the $\mathrm{N}$ - and C-terminus of the RBD are close to each other, which enables multimerization of RBDs through end-to- 
end connections without serious steric clashes. (2) There exist long flexible loops at both termini of the RBD, which would facilitate the connection of these different RBDs without destroying the core structure of the individual domain. (3) The tri-RBD construction strategy enables the realization of multivalent vaccine via a hybrid connection of RBDs from different circulating strains of SARS-CoV-2 into a single immunogenic molecule.

From the native tertiary structure of S protein, a RBD truncation scheme that meets the above considerations was designed, as shown in Supplementary Fig. S1a-c. The truncation scheme comprises the residues 319-537, which contains the entire RBD with long loops at both the $\mathrm{N}$ - and $\mathrm{C}$ - termini. Besides that, according to the immune-escape capability and evolutionary convergence of the mutations carried by SARS$\mathrm{CoV}-2$ variants, the RBDs from the prototype, Beta, and Kappa strains, respectively, were selected for the construction of the hybrid immunogen. Compared with the prototype strain, the RBD of Beta variant harbors three residue mutations, i.e., K417N, E484K, and N501Y, and the RBD of Kappa contains L452R and E484Q mutations, respectively. Several of these mutations were also revealed to be evolutionarily convergent. Three truncated RBDs were then connected end-to-end to generate a heterologous tri-RBD, i.e., mutI-tri-RBD. The homo-tri-RBD constructed only based on the prototype strain was also produced as a comparison (Fig. 1a).

The possible tertiary structures of the designed mutItri-RBD and homo-tri-RBDs were built by the Modeller9.23 software $^{25}$, using the native structure of $\mathrm{S}$ trimer as the template. To analyze the stereo-chemical rationality and stability of the modeled structures, the constructed trimeric structures were subjected to $200 \mathrm{~ns}$ all-atomic MD simulations with GROMACS software $^{26}$. Simulation results showed that both modeled mutI-tri-RBD and homo-tri-RBD structures were stereochemically stable without serious steric clashes. After 200 ns MD simulations, three RBDs were arranged in a loosely-packed trimeric form both in mutI-tri-RBD and homo-tri-RBD (Fig. 1b and Supplementary Videos S1 and S2). It should be mentioned that we do not exclude the possibility that the connected three RBDs assembled into another trimeric form different from that in the native structure of $\mathrm{S}$ trimer, which needs further validation by the experimental determination of its three-dimensional structure in future research.

\section{Expression, purification, and characterization of the recombinant mutl-tri-RBD and homo-tri-RBD}

The designed mutI-tri-RBD as well as homo-tri-RBD proteins of SARS-CoV-2 were transiently expressed in mammalian HEK293T cells. The culture supernatant of the transfected cells was harvested and purified by chromatography followed by ultrafiltration. Sodium dodecyl sulfate-polyacrylamide gel electrophoresis (SDS-PAGE) exhibited an obvious single band with the molecular mass of about $90 \mathrm{kDa}$ both for the recombinant mutI-tri-RBD and homo-tri-RBD proteins (Fig. 1c), indicating the integrity of the trimeric RBDs for the designed two schemes. Protein purity was determined over $95 \%$ for both mutI-tri-RBD and homo-tri-RBD. Furthermore, for mutI-tri-RBD protein, the exact molecular weight was determined to be around $87,836 \mathrm{Da}$ by matrix-assisted laser desorption ionization-time-of-flight mass spectrometer (MALDITOF MS) (Fig. 1d), which is larger than the theoretical value of about $74 \mathrm{kD}$ calculated based on amino acid sequence. The difference between the experimental and calculated molecular weights is attributed to the dense glycosylation of the protein. After removing the glycans, the molecular weight of the recombinant mutI-tri-RBD was determined to be $73,929 \mathrm{Da}$ by ultra-high performance liquid chromatography-mass spectrometer (UPLC-MS), which agrees well with the theoretical value (Fig. 1e).

Secondary structural composition analysis of the mutItri-RBD protein by circular dichroism (CD) illustrated that the recombinant protein was correctly folded with about $13.5 \%$ helix, $23.3 \% \beta$ strands, $11.4 \%$ turn, and $51.8 \%$ others (Fig. 1f and Supplementary Fig. S2a, b). For comparison, the secondary structures in an RBD monomer were further calculated by DSSP software ${ }^{27,28}$ based on the X-ray resolved structure (PDB accession code: $6 \mathrm{~m} 0 \mathrm{j})^{29}$, showing a composition of $10.1 \%, 22.4 \%, 8.7 \%$, and $58.8 \%$ for helix, $\beta$ strands, turn, and others, respectively. The sequence of one RBD contains 8 cysteine residues, and in the native structure of RBD in S protein, these cysteines form four intradomain disulfide bonds. Disulfide-linkages mapping by LC-MS showed that all these four disulfide bonds in RBD native structure also correctly formed in the recombinant mutI-tri-RBD protein (Fig. 1g and Supplementary Fig. S3a, b). The secondary structure and disulfide bonds analyses suggested that each of the three RBDs in the recombinant mutI-triRBD protein was correctly folded into its native structure. Then the transition temperature $\left(T_{\mathrm{m}}\right)$ was determined to be $47.2{ }^{\circ} \mathrm{C}$ by differential scanning calorimetry (DSC), demonstrating the stability of mutI-tri-RBD (Fig. 1h).

The bioactivity of the recombinant tri-RBD proteins was assessed using two anti-RBD monoclonal neutralizing antibodies (nAbs), i.e., MM43 and R117, by enzymelinked immunosorbent assay (ELISA). MM43 can bind not only with the prototype RBD but also with the Beta and Kappa RBDs. High binding activities with MM43 were observed both for the recombinant mutI-tri-RBD and homo-tri-RBD proteins, indicating the formation of native conformation for individual RBD in the designed 


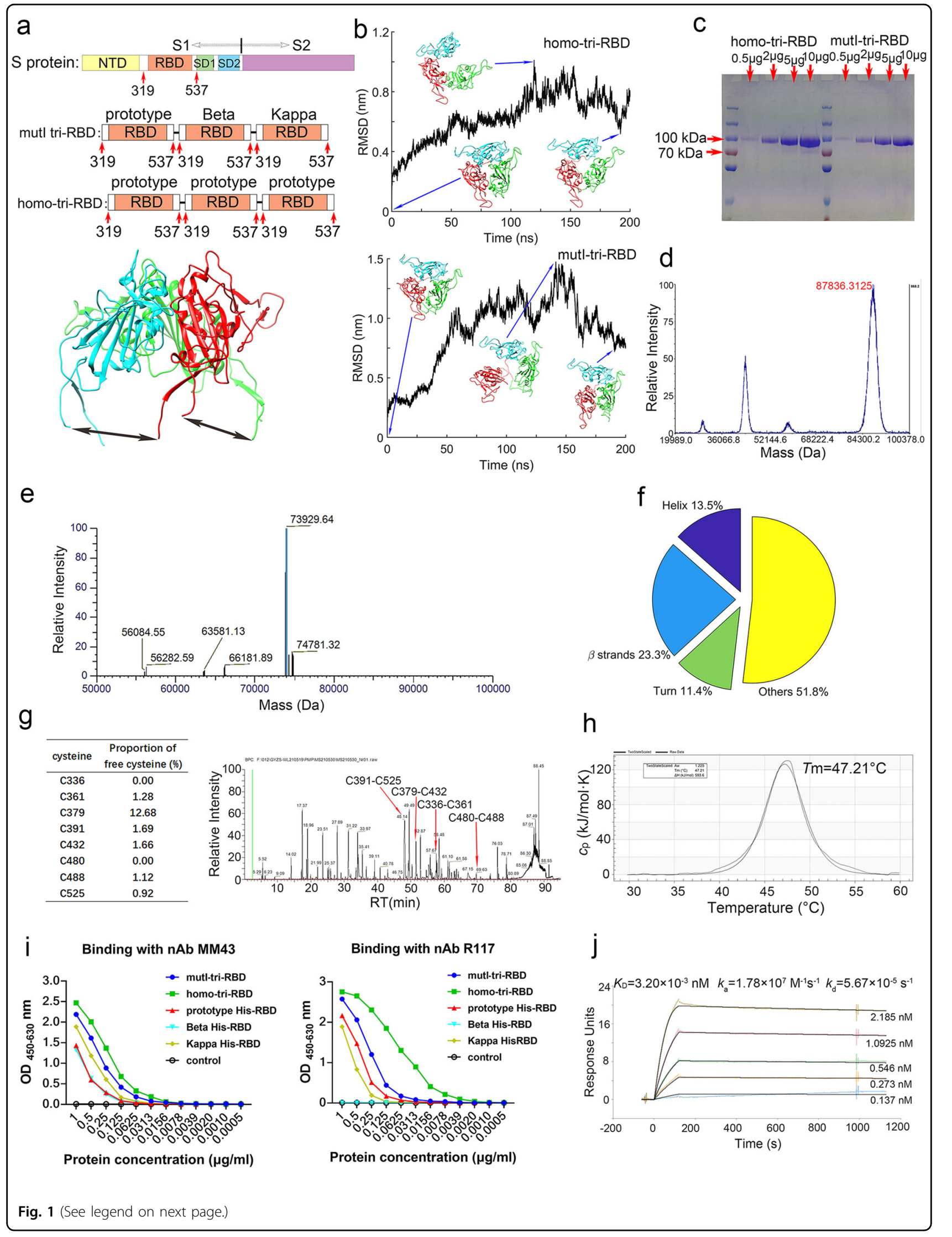


(see figure on previous page)

Fig. 1 Structure-guided design, production, and characterization of the mutl-tri-RBD. a A schematic illustration of the mutl-tri-RBD and homotri-RBD design schemes. The RBD region comprising the residues 319-537 was truncated from the S protein, and three truncated RBDs were connected end-by-end to construct the trimeric forms of RBD. In mutl-tri-RBD, three RBDs were individually derived from three different circulating SARS-CoV-2 strains, i.e., the prototype, Beta and Kappa. In homo-tri-RBD, the three RBD units were all truncated from the prototype strain. In the upper subfigure, the S1 and S2 subunits of the S protein, as well as NTD, RBD, SD1, and SD2 in the S1 subunit, are marked. The lower subfigure displays the natural trimeric arrangement of RBDs in the native structure of $\mathrm{S}$ trimer. The arrows indicate the direct connections of the $\mathrm{N}$ - and $\mathrm{C}$ - terminals between different RBDs. b Structural modeling and MD simulation of the designed homo-tri-RBD (upper subfigure) and mutl-tri-RBD (lower subfigure). Time-evolution of the $C_{a}$ root-mean square deviation (RMSD) of the modeled structure during MD simulation, as well as several snapshot conformations in the simulation, is displayed. c SDS-PAGE profiles of increasing amounts of the recombinant mutl-tri-RBD and homo-tri-RBD proteins expressed by HEK293T cells. d Molecular weight of mutl-tri-RBD determined by MALDI-TOF MS. e Molecular weight of mutl-tri-RBD after deglycosylation determined by UPLC-MS. f Secondary structure contents of mutl-tri-RBD protein analyzed by circular dichroism spectrometry. $\mathbf{g}$ Left: the proportions of free sulfhydryl for all the cysteine residues in mutl-tri-RBD. Right: the disulfide linkages in the recombinant mutl-tri-RBD protein detected by liquid chromatography-mass spectrometry. Only the disulfide bonds in one RBD unit are listed. $\mathbf{h}$ Differential scanning calorimetry thermograms of the recombinant mutl-tri-RBD protein. $\mathbf{i}$ The binding capability of the designed mutl-tri-RBD and homo-tri-RBD proteins with two anti-RBD monoclonal nAbs, i.e., MM43 and R117, evaluated by ELISA. As controls, the binding activities with the monoclonal nAbs for the monomeric his-tagged RBDs from the prototype, Beta, and Kappa SARS-CoV-2 strains were also measured. $\mathbf{j}$ The binding profiles of the recombinant mutl-tri-RBD with hACE2 detected by surface plasmon resonance assay.

trimeric proteins (Fig. 1i). R117 binds only to the prototype and Kappa RBDs, but not to the Beta RBD. A lower binding activity to R117 was observed in mutI-tri-RBD than that of homo-tri-RBD, especially at low concentrations (Fig. 1i), which is within our expectation as Beta RBD is designed to be embedded in mutI-tri-RBD.

Then, the binding strength of the mutI-tri-RBD protein with the receptor hACE2 was also quantified by surface plasmon resonance (SPR) assay. Owing to the avidity effect of the trimeric form of mutI-tri-RBD, the binding strength was evaluated by the apparent dissociation constant $K_{\mathrm{D}}$, which was determined to be $3.20 \times 10^{-3} \mathrm{nM}$, with the association rate constant $k_{\mathrm{a}}$ of $1.78 \times 10^{7} \mathrm{M}^{-1} \mathrm{~S}^{-1}$ and the dissociation rate constant $k_{\mathrm{d}}$ of $5.67 \times 10^{-5} \mathrm{~S}^{-1}$ (Fig. 1j). SPR assay demonstrated that the designed mutItri-RBD protein binds specifically to hACE2 with high avidity, implying correct folding of the RBDs and high functionality of the recombinant mutI-tri-RBD protein. These results supported the mutI-tri-RBD to serve as an excellent immunogen.

\section{Both mutl-tri-RBD and homo-tri-RBD elicited a high level of immune responses against the SARS-CoV-2 prototype strain in mice}

The immunological effects of the trimeric RBDs were tested in BALB/c mice using aluminum as the adjuvant. Animals were immunized intraperitoneally with two or three shots as shown in Fig. 2a, and for each shot, three different doses, including low-dose $(0.125 \mu \mathrm{g} / \mathrm{dose})$, middle-dose $(0.5 \mu \mathrm{g} / \mathrm{dose})$ and high-dose $(2.0 \mu \mathrm{g} / \mathrm{dose})$, respectively, were applied. The level of RBD-specific IgG in the mice sera, collected on day 7 post-immunization (D28 in Fig. 2a), was measured by using ELISA. RBD monomer from the SARS-CoV-2 prototype strain was used to coat the wells of the ELISA plates. Three-injection immunization regimen elicited distinctly higher immune response compared with the two-injection treatment, where the anti-RBD IgG GMTs were improved by 5.2, 4.0, and 3.0 times for the low-, middle- and high-dose vaccinations, respectively (Fig. 2b and Supplementary Table S1). Both in the two-shot and three-shot regimens, middle-dose immunization elicited a higher level of antiRBD IgG than low-dose, but no further increase was observed by high-dose vaccination (Fig. 2b and Supplementary Table S1). Furthermore, in order to compare the immunogenicity between mutI-tri-RBD and homo-tri$\mathrm{RBD}, \mathrm{BALB} / \mathrm{c}$ mice were also immunized with the homotri-RBD by two-injection of high dose or three-injection of middle dose. The results showed that the RBD-specific IgG induced by the mutI-tri-RBD was comparable to that elicited by the homo-tri-RBD, indicating similar immunogenicity between two trimeric RBDs (Fig. 2c and Supplementary Table S2).

Then, the GMT of the neutralizing antibodies against SARS-CoV-2 prototype strain was assessed by using the pseudo- and live virus neutralization assays. The sera from the immunized mice potently neutralized both the pseudo- and live virus infections. Three shots of the mutI-tri-RBD induced significantly higher neutralizing antibody titers than two shots, as shown by $6.2-36.8$ times higher against pseudo-virus and 6.2-34.9 times higher against live prototype virus (Fig. 2d, f and Supplementary Tables S3 and S5). Moreover, in animals immunized with the two-shot regimen, neutralizing GMT showed a dose-dependent increase in both pseudo- and live virus assays. In animals with the threeinjection regimen, middle dose induced the highest neutralizing antibody titer, and the neutralizing GMT against live prototype virus reached as high as 12,177 (Fig. 2f, g and Supplementary Tables S5 and S6). More importantly, both in the two-shot and three-shot immunizations, the GMTs of neutralizing antibodies 


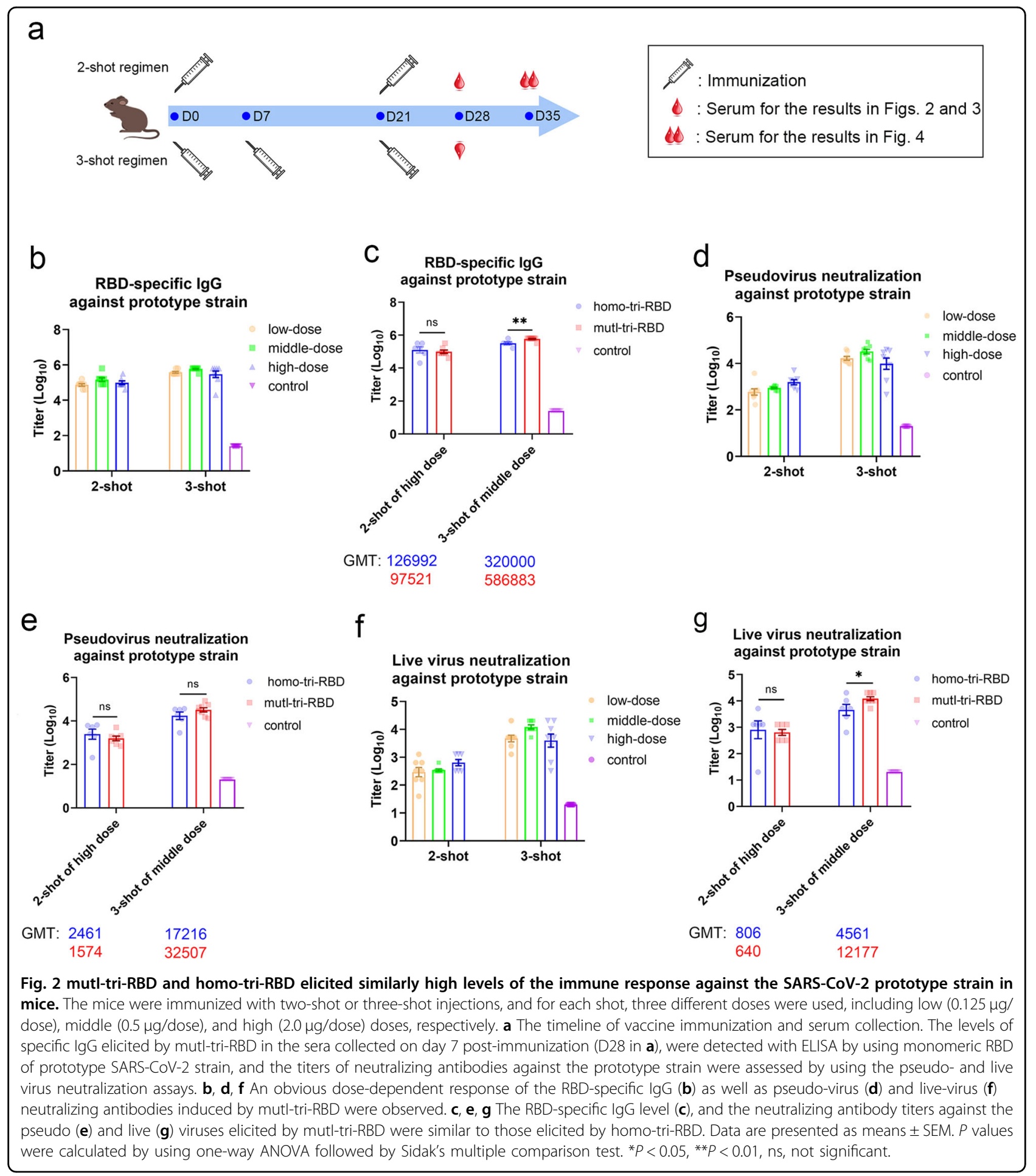

induced by mutI-tri-RBD were not less than those of homo-tri-RBD (Fig. 2e, g and Supplementary Tables S4 and S6). These results indicated that mutI-tri-RBD containing only one prototype RBD could elicit a similar response as homo-tri-RBD with three repeated prototype RBDs build-in.
The homo-tri-RBD has been approved by China National Medical Products Administration (NMPA) to enter a clinical trial. Here, the immune responses of IgG and neutralizing antibodies in mice suggested that the designed mutI-tri-RBD exhibited similar immunogenicity to the homo-tri-RBD against the SARS-CoV-2 prototype 
virus, although the former harbors only one-third prototype RBD in contrast to the latter. These results supported that the mutI-tri-RBD may also serve as an effective vaccine candidate against the SARS-CoV-2 prototype strain.

\section{mutl-tri-RBD induced significantly higher titer of neutralizing antibody responses against the Delta and Beta variants than homo-tri-RBD}

We hypothesized that mutI-tri-RBD would be a vaccine candidate with broad neutralizing effects against SARS$\mathrm{CoV}-2$ variants. Delta, as a highly transmissible variant, is rapidly becoming the dominant strain in many countries and also exhibits reduced sensitivity to neutralizing antibodies present in convalescent patients and vaccinated individuals. The Beta variant has also been believed to possess the ability to evade pre-existing immunity elicited by natural infections and vaccinations. Therefore, we first tested whether the designed mutI-tri-RBD can elicit robust neutralizing antibody responses against these two variants. The titers of neutralizing antibodies against the Delta and Beta variants, induced by the mutI-tri-RBD were evaluated in the mice sera, collected on day 7 postimmunization (D28 in Fig. 2a), by using pseudo- and live virus neutralization assays, which were then compared with those induced by the homo-tri-RBD.

Regarding the Delta variant, both mutI-tri-RBD and homo-tri-RBD induced elevated neutralizing antibody responses in the immunized mice; however, the neutralizing antibody levels stimulated by mutI-tri-RBD were significantly higher than those elicited by homo-tri-RBD both in pseudotype and live virus neutralization assays. The neutralizing antibody GMTs against the Delta pseudo-virus induced by mutI-tri-RBD were 3.0 -fold and 4.2-fold higher than those induced by homo-tri-RBD in the two-shot and three-shot injections, respectively (Fig. 3a and Supplementary Table S7). Correspondingly, in the live virus neutralization tests, the anti-Delta neutralizing GMTs were improved by 2.8 -fold and 6.4-fold, respectively, in the two-shot and three-shot vaccinations (Fig. 3b and Supplementary Table S8) for mutI-tri-RBD compared with those of homo-tri-RBD. Therefore, the mutI-tri-RBD exhibited superior immune efficacy than the homo-triRBD against the Delta variant.

Similar results were observed for neutralization assays on the Beta variant. Both two trimeric RBDs induced elevated neutralizing antibody responses; however, mutItri-RBD elicited significantly stronger neutralizing antibody responses against the Beta variant than homo-triRBD. The neutralizing GMTs against the pseudo-virus of Beta induced by mutI-tri-RBD were 3.4-fold and 2.7-fold higher compared with those induced by homo-tri-RBD in the two-shot and three-shot immunizations, respectively (Fig. 3c and Supplementary Table S9). The live virus neutralization assays further confirmed the pseudo-virus neutralization results, in which the neutralizing GMTs improved 3.0-fold and 5.3-fold, respectively, in the twoshot and three-shot vaccinations of the mutI-tri-RBD than those of the homo-tri-RBD (Fig. 3d and Supplementary Table S10). Particularly, a very high level of neutralizing antibody GMT of 17,222 against live Beta virus was observed in animals given with three-shots of mutI-tri-RBD (Fig. 3d and Supplementary Table S10). Thus, regarding the Beta variant, the immune efficiency of mutI-tri-RBD is distinctly better than that of homo-triRBD.

The above results demonstrated that the designed mutItri-RBD, which harbored RBD antigens from three circulating SARS-CoV-2 strains, not only maintained strong neutralizing antibody responses against the SARS-CoV-2 prototype strain but also induced significantly enhanced neutralizing antibody titers against the Delta and Beta variants than homo-tri-RBD.

\section{mutl-tri-RBD elicited broadly neutralizing activities against SARS-CoV-2 compared with homo-tri-RBD}

Besides the SARS-CoV-2 prototype, Delta, and Beta strains, we also investigated whether the designed mutItri-RBD can also elicit broad neutralizing antibody responses against other emerging SARS-CoV-2 variants. The pseudo-viruses of nine other circulating strains, including D614G, 501Y.V2-1, 501Y.V2-3, Alpha (B.1.1.7), Gamma (P.1), Zeta (P.2), Epsilon (B.1.429B), Eta (B.1.525), and Iota (B.1.526), as well as 11 corresponding single or combinatorial mutants were tested ${ }^{30-33}$, in pseudo-virus neutralization assays. Due to a large amount of serum needed, serum samples were re-collected on day 14 postvaccination (D35 in Fig. 2a) from the mice immunized with two shots of high dose of mutI-tri-RBD or homo-triRBD. The prototype, Delta, and Beta strains were also retested by using the 14-day post-immunization sera. In order to assess whether the mutI-tri-RBD has a superior effect in inducing broad neutralization against these SARS-CoV-2 strains than the homo-tri-RBD, the GMT values elicited by the mutI-tri-RBD were compared with those induced by the homo-tri-RBD.

We first examined whether the 22 tested SARS-CoV-2 variants exhibited resistance to the neutralization elicited by homo-tri-RBD. Neutralization experiments showed that although homo-tri-RBD can induce neutralizing antibodies against all these tested variants in comparison with the control group, some variants also displayed obviously reduced sensitivities to the neutralization of the immunized sera compared with the prototype strain. Among the eleven circulating variants, Delta, Beta, Alpha, Zeta, Epsilon, Eta, and Iota exhibited significantly decreased neutralization susceptibilities by varying extents (Fig. 4a). Notably, all these variants harbored 
a

\section{Pseudovirus neutralization against Delta strain}

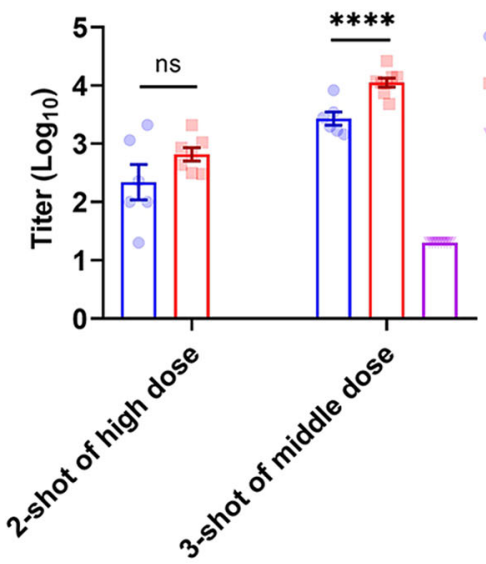

GMT: 219 657

C

\section{Pseudovirus neutralization against Beta strain}
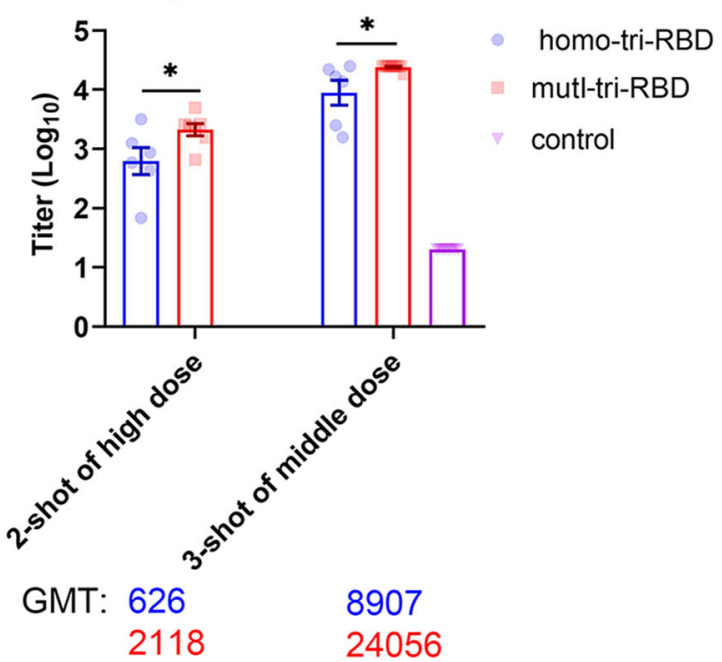

b
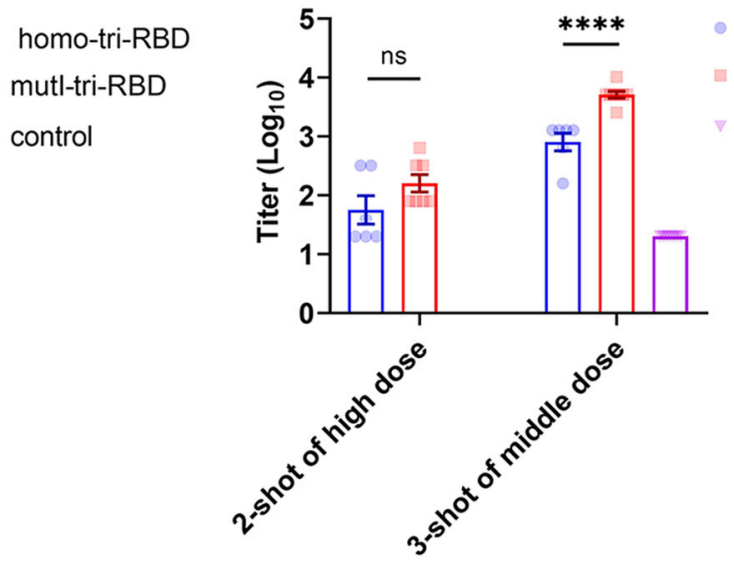

GMT: 57

806

160
homo-tri-RBD

mutl-tri-RBD

control

d

\section{Live virus neutralization against Beta strain}
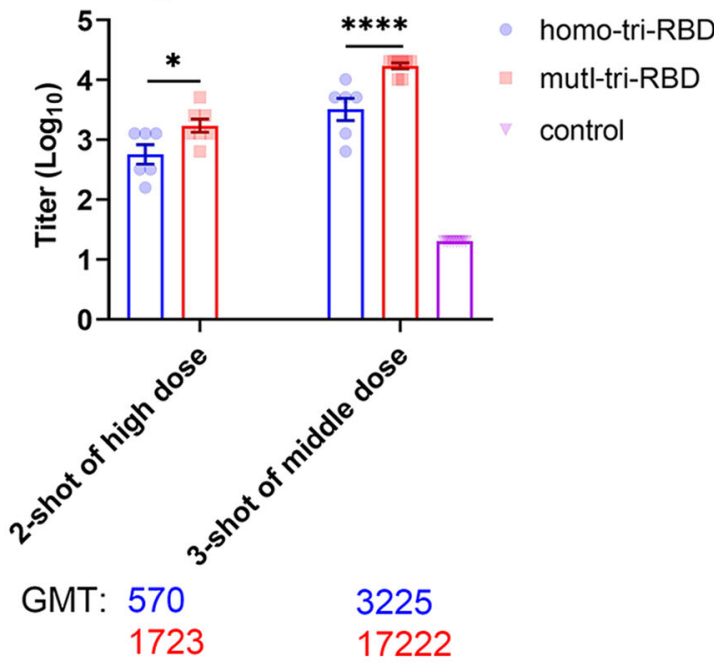

Fig. 3 mutl-tri-RBD induced significantly higher titer of neutralizing antibody responses against the Delta and Beta variants compared with homo-tri-RBD. a, b The pseudo-virus (a) and live-virus (b) neutralizing antibody titers against the SARS-CoV-2 Delta variant induced by mutl-triRBD in the sera collected on day 7 post-immunization (D28 in Fig. 2a) were distinctly higher than those elicited by homo-tri-RBD. $\mathbf{c}$, $\mathbf{d}$ The pseudovirus (c) and live-virus (d) neutralizing antibody titers against SARS-CoV-2 Beta variant induced by mutl-tri-RBD were also obviously higher than those elicited by homo-tri-RBD. Data are presented as means \pm SEM. P values were calculated by using one-way ANOVA followed by Sidak's multiple comparison test. ${ }^{*} P<0.05,{ }^{* * *} P<0.001$, ns, not significant.

E484K, L452R, or N501Y mutation, suggesting the importance of these residues for immune resistance. This is further demonstrated by the corresponding single and combinatorial mutants of E484K, L452R, or N501Y, as most of them also displayed distinctly reduced neutralization sensitivities (Fig. 4a). In addition, the single mutation of E484Q also exhibited obviously reduced neutralization sensitivities to the antibodies elicited by
homo-tri-RBD. However, the neutralization reactions against the $\mathrm{K} 417 \mathrm{~N}$ and $\mathrm{T} 478 \mathrm{~K}$ single-mutants were not reduced, implying that these two residues do not lead to immune resistance (Fig. 4a). This is consistent with the experimental results from another work, where K417N mutation leads to enhanced neutralization activity ${ }^{31}$.

Then, we tested whether the designed mutI-tri-RBD can induce more robust neutralization activities against these 


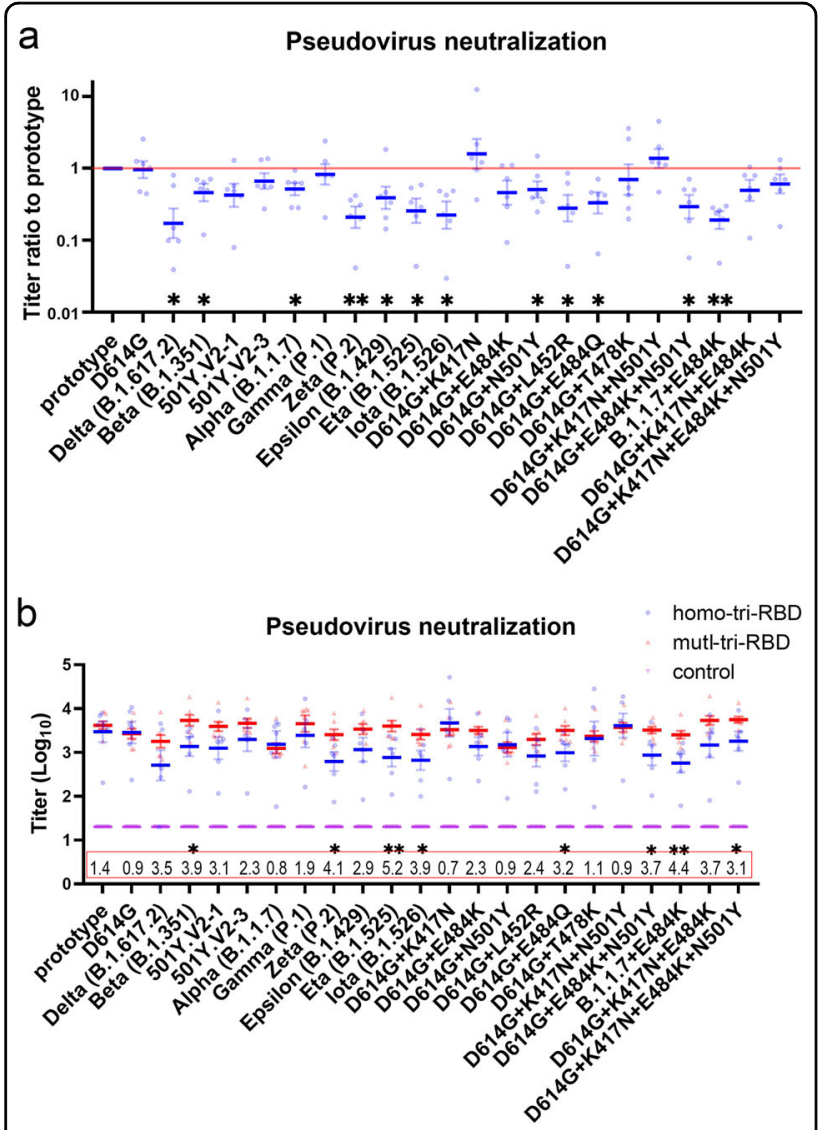

Fig. 4 The neutralizing antibody responses against 23 various SARS-CoV-2 pseudo-virus strains elicited by mutl-tri-RBD were significantly stronger than or comparable to those induced by homo-tri-RBD. a Sensitivities to the neutralization of homo-tri-RBD immunized sera for the various pseudo-typed variants compared with that of the prototype strain. The sera collected on day 14 post-

immunization (D35 in Fig. 2a) were used in the pseudo-virus neutralization assays. In this figure, the titers of the prototype strain are taken as a reference, and the titer ratios between the variants and the prototype strain are displayed. Each serum sample is presented as a dot in the plot, and each serum sample was tested against all these variants. Data are presented as means \pm SEM. $P$ values were calculated with Student's $t$ test. ${ }^{*} P<0.05$, ${ }^{*} P<0.01$. b Neutralizing antibody GMTs against the various pseudo-typed strains induced by mutl-triRBD (red color) compared with those elicited by homo-tri-RBD (blue color). Numbers in the red box indicate the GMT ratios of mutl-tri-RBD to homo-tri-RBD. Data are presented as means \pm SEM. $P$ values were calculated by using one-way ANOVA followed by Sidak's multiple comparison test. ${ }^{*} P<0.05,{ }^{*} P<0.01$.

22 SARS-CoV-2 variants than homo-tri-RBD, especially the homo-tri-RBD-resistant variants discussed above. The results showed that in almost all tested strains, neutralization GMTs induced by mutI-tri-RBD were comparable to or significantly higher than those induced by homo-tri-RBD. For E484K- or L452R-carrying variants, including Delta, Beta, 501Y.V2-1, 501Y.V2-3, Gamma, Zeta, Epsilon, Eta, and Iota, the neutralization activities induced by mutI-tri-RBD were improved by 1.9 to 5.2 -fold in contrast to those induced by homo-tri-RBD (Fig. 4b and Supplementary Information, Table S11). For the E484K, L452R, as well as E484Q single mutations, the neutralization sensitivities elicited by the mutI-tri-RBD were raised 2.3-, 2.4-, and 3.2-fold, respectively, (Fig. 4b and Supplementary Table S11). Most importantly, as discussed above, the E484K, L452R, and E484Q mutations lead to obvious resistance to the sera immunized with homo-tri-RBD, which may partly reduce the efficacy of the homo-tri-RBD vaccine candidate. However, encouragingly, the mutI-tri-RBD enables a substantial increase of the neutralizing titers against these homo-tri-RBDresistant variants, indicating potently broad neutralizing capabilities induced by mutI-tri-RBD (Fig. $4 \mathrm{~b}$ and Supplementary Table S11). E484K has been believed to be the most important "immune escape" mutation, and our pseudo-virus neutralization assays displayed that the mutI-tri-RBD also induced robustly higher neutralizing antibody responses against the E484K-carrying combinatorial mutants in comparison with the homo-tri-RBD.

Many studies have demonstrated that the neutralizing antibody level is highly correlated with the protective efficacy, in which the higher the neutralizing antibody titer is, the lower the chance of breakthrough infection 34-37. Therefore, the neutralizing antibody titer against different SARS-CoV-2 variants is a crucial indicator to evaluate the broadly protective ability of the vaccine. Our experimental results show that compared with homo-triRBD, mutI-tri-RBD elicited much higher neutralizing antibody titers against most of the tested SARS-CoV-2 variants, which indicates that it may potentially serve as a more promising broad-spectrum vaccine candidate against SARS-CoV-2 for further clinical developments.

\section{mutl-tri-RBD efficiently protected hACE2-transgenic mice from challenges with the SARS-CoV-2 prototype, Delta, and Beta live viruses}

In order to validate the broad-spectrum protective ability of the designed mutI-tri-RBD in vivo, its protective efficacy was assessed in hACE2-transgenic mice against live virus challenges with three major SARS-CoV-2 strains, including the prototype, Delta and Beta. The mice in vaccine groups were immunized with two doses of mutI-tri-RBD with $2 \mu \mathrm{g} /$ dose on Day 0 and Day 21, and correspondingly the mice in the saline groups and control groups were treated with two doses of physiological saline. On Day 7 after the whole vaccination, high titers of neutralizing antibodies were detected for all mice in vaccine groups, whereas in the corresponding saline groups, no neutralizing activity was observed (Fig. 5a and Supplementary Table S12). On day 14 or day 19 after the complete immunization, the mice in vaccine groups and saline groups were challenged with the live virus of the prototype, Delta and Beta SARS-CoV-2 strains, 


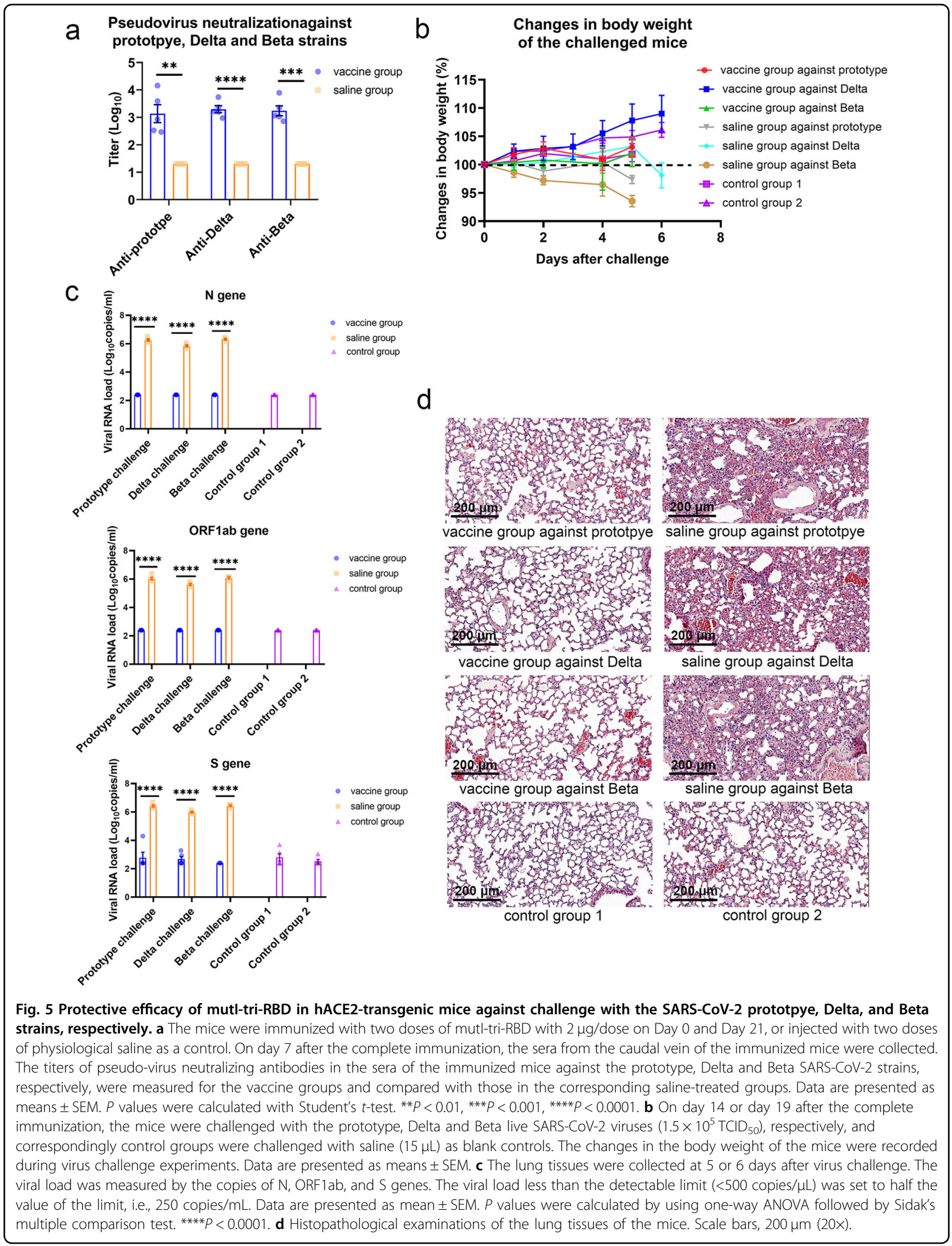


respectively. The mice in control groups were challenged with saline as blank controls. During the live virus challenge experiment, a mouse in the saline group challenged with Beta strain died on the fifth day. All the other mice survived, but muscle weakness and vitality decrease began to appear from the third day for the mice in all the saline groups challenged with the prototype, Delta or Beta strains. Especially, the mice in the saline group challenged with Beta virus experienced an obviously progressive drop in body weight. For the mice in all the vaccine groups, no infection-related symptoms and body weight loss were observed when compared with the control group (Fig. 5b and Supplementary Table S13).

Viral load in mice lung tissues was detected qPCR of N, ORF1ab, and S genes. All animals in saline groups showed high viral RNA loads after the live virus challenge. On the contrary, negative $(<500$ copies $/ \mathrm{mL})$ or very low viral loads were monitored in lung tissues for all the mice in the vaccine groups challenged with the prototype, Delta or Beta viruses (Fig. 5c and Supplementary Table S14). Histopathological examination of the lung tissues showed that severe interstitial pneumonia, a typical feature of COVID-19, occurred in the mice of the saline groups. Substantial histopathological changes were observed, including alveolar septa thickening, heavy inflammatory cell infiltration, and focal hemorrhage. Large amounts of exudates appeared in lung cavities, as well as the formation of hyaline membrane and bullae were also observed. In addition, tracheal bronchus and blood vessels also exhibited a severe infiltration and exudates combined with desquamation of epithelial cells. In contrast, no obvious histopathological change was observed in the lung tissues of the mice in all the vaccine groups challenged with the prototype, Delta or Beta SARS-CoV-2 viruses. The mice receiving vaccination showed normal lung tissue with only focal mild inflammatory infiltrations, which were similar to the mice in the control groups but significantly different from the results in the above saline groups (Fig. 5d). The animal challenge experiments indicated that mutI-tri-RBD vaccine candidate provided efficient broad protection not only against the prototype SARS-CoV-2 virus but also against the Delta and Beta variants.

\section{Discussion}

RBD is directly involved in the recognition of SARSCoV-2 to host cells, which is immunodominant in eliciting neutralizing antibody responses. As of October 2021, at least 23 RBD-based vaccine candidates are under clinical trials, among which a dimeric RBD-based protein subunit vaccine, ZF2001, has been authorized for emergency use ${ }^{38}$. Preclinical and clinical data revealed that RBD-based immunogen was not inferior to the fulllength $\mathrm{S}$ protein in immunicity ${ }^{39}$. Clinical trial results showed that the RBD-dimer ZF2001 vaccine induced twofold higher neutralizing antibody GMTs than that of human convalescent sera $(\mathrm{HCS})^{40}$, and the neutralizing GMTs yielded by the RBD-based nanoparticle vaccines were five times higher than $\mathrm{HCS}^{41}$. For the homo-triRBD vaccine candidate developed by our group, the preliminary results in the phase I/II clinical trial showed that neutralizing antibody GMTs were about 3-5-fold higher than those of HCS (data not yet published). Although RBD-based vaccines exhibited relatively robust and broad immunogenicity, the neutralizing efficacy against some of the SARS-CoV-2 variants was declined to a certain extent ${ }^{42,43}$.

The new waves of COVID-19 caused by SARS-CoV-2 variants and the rising number of breakthrough infections highlight the need of developing broadly protective vaccines to combat the present and future SARS-CoV-2 variants. Naturally trimeric arrangement of RBDs in $\mathrm{S}$ protein and its unique structural characteristics allow construction of heterogenous mutI-tri-RBD as a broadly protective vaccine candidate, in which the RBD antigens derived from different SARS-CoV-2 circulating strains were integrated into a single immunogen. The designed mutI-tri-RBD induced broadly neutralizing antibody responses against various circulating SARS-CoV-2 strains. Compared with the homo-tri-RBD, the hybrid mutI-triRBD displayed broader and superior neutralization efficacy against SARS-CoV-2. The broadly protective ability of mutI-tri-RBD was validated by using live virus challenge experiments, in which it not only potently protected the animals from the infections of the SARS-CoV-2 prototype strain but also provided efficient protection against the highly infectious Delta and the so-called "immune escape" Beta variants.

The anti-Beta vaccine candidate has recently been tested for a bivalent vaccination strategy in combination with the anti-prototype one or for heterologous prime boosting immunization strategy ${ }^{44-46}$. Here, we provided another promising strategy for the construction of a vaccine candidate with broad neutralizing capability by incorporating different variant-specific antigens and key mutations into one hybrid immunogen. Compared with the conventional multivalent strategy, our method has the advantages of broader protection capability, lower time costs, and higher production benefits.

Evolutionary analysis indicated that most of the immune-escape mutations integrated into mutI-tri-RBD are also evolutionarily convergent and considered to be of adaptive advantage, suggesting the possible recurrence of them individually or combinedly in the future ${ }^{14}$. Our results demonstrated that the designed mutI-tri-RBD induced a broader and robust neutralizing response against these evolutionarily-convergent and immuneresistant mutations, suggesting that our hybrid 
immunogen construct may have the potential to provide neutralizing capability against future-emerging SARSCoV-2 variants.

Hybrid immunogen design strategy was also adopted by other groups to develop broadly protective vaccines against SARS-CoV-2 variants and other coronaviruses. Cohen et al. ${ }^{47}$ and Walls et al. ${ }^{48}$ constructed mosaic nanoparticle immunogens, in which multivalent RBDs from various coronavirus were co-displayed on the same particle surface. Owing to the high-dense and heterotypic arrays of the antigens, this mosaic nanoparticle-type vaccine candidate elicited much stronger and broader antibody responses compared with the monomeric immunogen. It was also immunogenically superior to the homotypic RBD nanoparticles. However, it is challenging for precise control of the relative number and distribution of different RBDs coated on the nanoparticle surface. In addition, the nanoparticle scaffold might also have unknown safety risks. Martinez et al. ${ }^{15}$ designed a chimeric $S$ protein immunogen, in which the NTD, RBD, and S2 domains derived from different sarbecoviruses were integrated into a single molecule. Although the chimeric immunogen induced broadly protective antibodies against diverse sarbecoviruses, the construction strategy is not applicable for the assembly of different RBDs into a single immunogen. Compared with these approaches, the mutItri-RBD designed by our group enables the integration of multiple immunodominant RBDs into a single immunogen to improve and broaden immunogenicity. In our method, no exogenous sequence was introduced, which ensured the safety profile of this vaccine candidate.

Structure-guided design of new immunogen that integrates key immune-relevant mutations is an effective strategy for the development of vaccines with broadly protective capabilities, which can be used for other highly variable viruses besides SARS-CoV-2. This mutation-integration strategy can be realized not only in protein subunit vaccine developments but also in mRNA and DNA vaccine platforms.

After we have submitted the manuscript, during the review process, SARS-CoV-2 Omicron is growing rapidly to be the most prevalent variant. A randomized clinical trial for mutI-tri-RBD has been conducted in the United Arab Emirates, and the neutralizing activity against Omicron variant induced by the vaccine will be evaluated in the trial. Currently, the trial is still ongoing, and we will report the data in the near future.

\section{Materials and methods}

\section{Structure modeling and MD simulations}

To construct mutI-tri-RBD, the RBD region (residues 319-537) was truncated from the $S$ proteins of the prototype, Beta (B.1.351) and Kappa (B.1.617.1) SARS$\mathrm{CoV}-2$ strains, respectively. Then these three RBDs were connected end-to-end by using their own long loops at the $\mathrm{N}$ - and $\mathrm{C}$-terminus without introducing exogenous linker. As a comparative control, the homo-tri-RBD was also constructed in which the three RBDs were all derived from the prototype strain. The possible threedimensional structures of the designed mutI-tri-RBD and homo-tri-RBD proteins were modeled with the Modeller9.23 software ${ }^{25}$ by using the native structure of $\mathrm{S}$ trimer (PDB accession code 6zgi for homo-tri-RBD, as well as 6zgi and 7lyl for mutI-tri-RBD) as the template. A total of 10 structures were generated both for mutI-triRBD and homo-tri-RBD, and the structure with the lowest value of the DOPE assessment score ${ }^{49}$ was picked as the best model.

Then, a $200 \mathrm{~ns}$ atomic MD simulation was performed both for the mutI-tri-RBD and homo-tri-RBD. All the MD simulations were carried out by using Gromacs 2019 with the Charmm27 force field ${ }^{26}$. The trimeric structure generated by Modeller ${ }^{25}$ was solvated using SPC water molecules in a cubic box, with the protein atoms being at least $1.6 \mathrm{~nm}$ away from the box edges. A total of 24 and $21 \mathrm{CL}^{-}$ions were added into the water box to neutralize the net charges of the simulation systems for the mutItri-RBD and homo-tri-RBD, respectively. The prepared system was then subjected to energy minimization with the steepest descent algorithm to ensure that the maximum force in the system was below $1000 \mathrm{~kJ} / \mathrm{mol} / \mathrm{nm}$. After energy minimization, a $100 \mathrm{ps}$ NVT simulation with position restraints on protein atoms was performed at $300 \mathrm{~K}$, which was followed by a $100 \mathrm{ps}$ NPT simulation with position restraints. Finally, the production simulation without any position restraint was run for $200 \mathrm{~ns}$ and the snapshots were collected every $10 \mathrm{ps}$ to obtain the simulation trajectory. In the simulation, a time step of $2 \mathrm{fs}$ was used and all $\mathrm{H}$-bonds were constrained with LINCS algorithm. Both in the mutI-tri-RBD and homotri-RBD, each RBD unit contains four disulfide bonds, and these disulfide bonds were included in the construction of the topology files for the simulation systems. A cutoff value of $1.0 \mathrm{~nm}$ was used for the calculation of both short-range electrostatic and van der Waals interactions. Long-range electrostatic interactions were computed by using the Particle-Mesh Ewald (PME) algorithm. During the simulation, the temperature and the pressure of the system were maintained at $300 \mathrm{~K}$ and 1 bar with the velocity rescaling and the isotropic Parrinello-Rahman coupling methods, respectively. Based on the MD simulation trajectories, the changes in the root mean square deviation of the $C_{\alpha}$ atoms of the system as a function of time were calculated by using the built-in tool of "gmx rms" in Gromacs to evaluate the stability of the trimeric RBD proteins, and the motion movies were generated by using Chimera software ${ }^{50}$ to display the intrinsic conformational movements of the trimeric RBD proteins. 


\section{Protein expression and purification}

The gene sequences of the designed mutI-tri-RBD and homo-tri-RBD proteins were codon optimized for the transient expression in the mammalian cells. In the designed trimeric schemes, mutI-tri-RBD is composed of three $\mathrm{RBD}$ regions derived from the $\mathrm{S}$ proteins of the prototype, Beta and Kappa SARS-CoV-2 strains, respectively, connected end-to-end with each other. Homo-triRBD consists of three copies of the RBD region from the prototype strain. For gene cloning of these designed proteins, signal peptide and Kozak sequences were added to the $\mathrm{N}$-terminal of the expressed protein sequences, which were then inserted into the PTT5 plasmid via the HindIII and NotI restriction sites to construct the recombinant plasmids for the expression of the trimeric RBDs in HEK293T cells. The sequences of the constructed plasmids for the mutI-tri-RBD and homo-tri$\mathrm{RBD}$ proteins were verified by gene sequencing. Then, the generated plasmids were transfected into the HEK293T cells for transient expression. After 3-5 days of culture, the culture supernatants from the transfected cells were harvested and purified by chromatography. During the chromatographic purifications, the isolated proteins from the eluted peaks were analyzed with SDSPAGE. Then, the recovered protein sample was finally purified by ultrafiltration with the membranes of $30 \mathrm{kDa}$ molecular weight cutoff. The purity of the produced proteins was determined by the size exclusion chromatography-high-performance liquid chromatography (SEC-HPLC) using TSKgel G2500PW column.

\section{Protein molecular weight measurement}

MALDI-TOF-MS analysis was carried out with $A B$ Sciex 4800 Plus MALDI TOF analyzer to measure the molecular weight of the recombinant mutI-tri-RBD ${ }^{51,52}$. The protein sample was exchanged into the water to the concentration of $5 \mathrm{mg} / \mathrm{mL}$, and $0.5 \mu \mathrm{L}$ of the sample was spotted onto the MALDI target plate, followed by air drying at room temperature. The MALDI plate was then covered with $0.5 \mu \mathrm{L}$ of $0.5 \mathrm{mg} / \mathrm{mL}$ sinapinic acid (SA) dissolved in $0.1 \%$ TFA and $50 \%$ CAN, and also dried by air at room temperature. The MALDI TOF/TOF analyzer was equipped with a Nd:YAG $355 \mathrm{~nm}$ laser. Parameters were set as follows: linear mode; repetition rate laser: $200 \mathrm{~Hz}$; mass range: $20,000-150,000 \mathrm{Da} ; 400$ shots accumulated per profile.

\section{Measurement of protein molecular weight after deglycosylation}

Firstly, the $\mathrm{N}$ - and O-linked glycans were removed from the protein samples. $100 \mu \mathrm{g}$ of the sample was mixed with $5 \mu \mathrm{L} 10 \times$ Deglycosylation Mix Buffer 1 (B6044S) and $5 \mu \mathrm{L}$ Protein Deglycosylation Mix II (P6044S), which was then incubated at the room temperature for $30 \mathrm{~min}$ and further at $37^{\circ} \mathrm{C}$ for $16 \mathrm{~h}$. After that, the fully deglycosylated protein was separated by UPLC with Thermo Fisher Scientific UltiMate 3000 system. UHPLC was performed using a NanoMico UniPS 3-300 Column $(3 \mu \mathrm{m}, 2.1 \mathrm{~mm} \times 50 \mathrm{~mm})$ with a mobile phase of $0.1 \%$ formic acid (FA)-water and $0.1 \%$ FA-acetonitrile. The temperatures of the sample chamber and the column were respectively maintained at 4 and $60^{\circ} \mathrm{C}$, and the flow rate was set to $0.4 \mathrm{~mL} / \mathrm{min}$. Then, the eluting sample was analyzed using Thermo Fisher Scientific Q Exactive Plus mass spectrometer (MS), in which the MS spectra were acquired in positive ion mode for $8 \mathrm{~min}$ with a scan range of $1000-4000 \mathrm{~m} / \mathrm{z}$ and a resolution of 17,500. The acquired raw data files were analyzed with BioPharma Finder software to calculate the molecular weight.

\section{Disulfide bond identification}

To identify the disulfide bond locations in the recombinant mutI-tri-RBD protein by using the UPLCMS peptide mapping method, both reduced and nonreduced protein samples were prepared. In sample preparation, the NEM solution was firstly added into the sample to block free sulfhydryl groups in the protein at room temperature for $2 \mathrm{~h}$. Then, the protein was transferred into a digestion buffer and pre-cleaved with Lys-C at $37^{\circ} \mathrm{C}$ for $4 \mathrm{~h}$ followed by additional digestion with Glu-C at $37^{\circ} \mathrm{C}$ overnight. Half of the digested sample was taken as the non-reduced sample, and the other half was further subjected to reduction and alkylation to prepare the reduced sample. In reduction and alkylation reactions, the digested peptides were reduced with DTT at $37^{\circ} \mathrm{C}$ for $30 \mathrm{~min}$ and alkylated with IAA in the dark at room temperature for an additional $30 \mathrm{~min}$. Then the resulted peptides were separated both for the reduced and non-reduced samples by UPLC using a C18 column (130, $1.7 \mu \mathrm{m}$, $2.1 \mathrm{~mm} \times 150 \mathrm{~mm}$ ) with the column temperature of $60^{\circ} \mathrm{C}$, and the reversed-phase chromatography of the sample was performed for $95 \mathrm{~min}$ with a flow rate of $0.3 \mathrm{~mL} / \mathrm{min}$ and a UV detection wavelength of $214 \mathrm{~nm}$. Subsequently, the separated peptides were detected and analyzed by Q Exactive Plus MS in positive ion mode, with $m / z$ range of 300-2000 and a resolution (Full MS/ MS2) of 35,000/17,500. The MS raw data files were processed using BioPharma Finder software (version 3.2). The presence of disulfide bonds containing peptides was determined through comparative analysis of the reduced and non-reduced peptide samples and were shown as indicated in the chromatogram as shaded peaks. The free sulfhydryl of a certain cysteine was quantified through the ratio of MS area of the NEM modification containing peptides against MS area of all peptides in the reduced sample. 


\section{Determination of secondary structural composition}

Protein secondary structural composition was determined by using $\mathrm{CD}$ spectroscopy, which was performed on Applied Photophysics following the operation procedure provided by the manufacturer. The protein sample was dialyzed into the buffer composed of $5 \mathrm{mM} \mathrm{PB}$, and then placed to the spectrophotometer cell. Both far-ultraviolet (UV) and nearUV spectra were acquired in the wavelength range of $190-250$ and $250-304 \mathrm{~nm}$, respectively, with a spectral resolution of $0.5 \mathrm{~nm}$ and bandwidth of $1.0 \mathrm{~nm}$. All CD spectra were obtained with $0.5 \mathrm{~S}$ per point at $25^{\circ} \mathrm{C}$. Both the spectra of the sample and the buffers under the same experimental conditions were recorded, and the actual protein spectra were obtained by subtracting the spectra of the buffers. The scan measurement was repeated 6 times and the average result was calculated to ensure the reliability of the measurement. The acquired $\mathrm{CD}$ data were analyzed with the BeStSel Server (http://bestsel.elte.hu/index.php) ${ }^{53}$ to estimate the content of different secondary structures in the protein.

\section{Protein stability characterization}

The thermal stability of the recombinant mutI-tri-RBD was characterized by using a DSC assay. The protein sample was prepared at the concentration of $2.0 \mathrm{mg} / \mathrm{mL}$ in origin formulation buffer and $0.3 \mathrm{~mL}$ of the sample was used to perform the DSC experiment with the TA Instrument Nano-DSC. The thermograms were obtained over the temperature range from 5 to $95^{\circ} \mathrm{C}$ using a scan rate of $1{ }^{\circ} \mathrm{C} /$ min. The experimental data were processed by Launch NanoAnalyze software supplied with the DSC instrument.

\section{SPR assay}

The binding avidity of mutI-tri-RBD with receptor hACE2 was measured by using surface plasmon resonance (SPR), which was carried out by BIAcore $8 \mathrm{~K}$ (GE Healthcare) with NTA chips. The His-tagged receptor protein hACE2 (Sino Biological Inc., China. Cat: 10108$\mathrm{H} 08 \mathrm{H}$ ) was dissolved in HBS-T buffer (HBS buffer and $0.05 \%$ Tween 20 ) and immobilized onto the NTA chip. The protein sample was diluted with HBS-T buffer at the concentrations of $0.0173,0.0346,0.0692,0.1385$, and $0.277 \mu \mathrm{g} / \mathrm{mL}$, which were then flowed over the chip surface at a rate of $30 \mu \mathrm{L} / \mathrm{min}$ for $120 \mathrm{~s}$. The bound protein was then dissociated for an additional $120 \mathrm{~s}$. During the association and dissociation processes, the real-time response SPR signal was recorded. After that, the sensor chip was regenerated using $350 \mathrm{mM}$ EDTA regeneration solution for $120 \mathrm{~s}$ with a flow rate of $30 \mu \mathrm{L} / \mathrm{min}$. The recorded data were processed using BIAcoreTM Insight Evaluation software and the binding kinetics were analyzed with a 1:1 binding model to obtain the association rate constant $k_{\mathrm{a}}$, the dissociation rate constant $k_{\mathrm{d}}$, and the apparent dissociation constant $K_{\mathrm{D}}$.

\section{Evaluation of $n A b$-binding activities}

The binding activities of the recombinant mutI-tri-RBD and homo-tri-RBD proteins with two monoclonal nAbs, including MM43 and R117 (Sino Biological Inc., China. Cat: 40591-MM43 and 40592-R117), were evaluated by using ELISA. As controls, the binding activities with the monoclonal nAbs for the monomeric his-tagged RBDs from the prototype, Beta (B.1.351) and Kappa (B.1.617.1) SARS-CoV-2 strains were also measured. To detect the binding activity with the anti-RBD monoclonal nAbs, the protein samples were prepared at the starting concentration of $1.0 \mu \mathrm{g} / \mathrm{mL}$, which were then subjected to 2-fold serial dilutions and coated on the 96-well microplate with $100 \mu \mathrm{L}$ per well at $2-8{ }^{\circ} \mathrm{C}$ overnight. Subsequently, the plate was washed 3 times with phosphatebuffered saline (PBS) containing 0.05\% Tween 20 (PBST) and then blocked with $100 \mu \mathrm{L}$ blocking buffer per well, followed by incubation at $37^{\circ} \mathrm{C}$ for $2 \mathrm{~h}$. After washing the plate 3 times with PBST, the nAb sample was diluted to $1.0 \mu \mathrm{g} / \mathrm{mL}$ and added into the wells with $100 \mu \mathrm{L}$ per well, which was then incubated at $37^{\circ} \mathrm{C}$ for $1 \mathrm{~h}$. After that, the plate was washed 3 times with PBST and the horseradish peroxidase (HRP)-conjugated goat anti-mouse or goat anti-rabbit IgGs at 1:10,000 dilution was added into the wells of the plate. The plate was again incubated at $37^{\circ} \mathrm{C}$ for $1 \mathrm{~h}$ and washed 3 times with PBST. Subsequently, $50 \mu \mathrm{L}$ tetramethylbenzidine (TMB) and $50 \mu \mathrm{L}$ hydrogen peroxide solutions were added to start the color reaction. After color development for $5 \mathrm{~min}$, the reaction was stopped using $0.2 \mathrm{M}$ sulfuric acidic solution with $50 \mu \mathrm{L}$ per well, and absorbance at $450 \mathrm{~nm}$ was read by plate reader. The background absorbance at $630 \mathrm{~nm}$ was also measured, and then the difference between absorbance at $450 \mathrm{~nm}$ and $630 \mathrm{~nm}\left(\mathrm{OD}_{450 / 630 \mathrm{~nm}}\right)$ was obtained to detect the specific binding of the mutI-tri-RBD and homo-triRBD proteins with the nAbs.

\section{Ethics statement}

All the animal experimental procedures were carried out according to Chinese animal use guidelines and were approved by the Institutional Animal Care and Use Committee (IACUC) of the National Vaccine and Serum Institute (NVSI) of China. All challenge experiments with live SARS-CoV-2 were approved by the IACUC of NVSI of China, and the IACUC of National Institute for Viral Disease Control and Prevention, Chinese Center for Disease Control and Prevention, Beijing, China.

\section{Mouse immunization protocols}

To evaluate the impacts of different immunization regimens as well as vaccination doses on the immune responses, a total of 6 groups of female specific pathogen-free (SPF) mice (purchased from Beijing Vital River Laboratory Animal Technology Co., Ltd., China.) 
with 8 mice in each group, aged 6-8 weeks, were intraperitoneally vaccinated with the mutI-tri-RBD vaccine candidate mixed with aluminum hydroxide adjuvant. In these experimental groups, the mice were immunized with two shots on day 0 and day 21 , or with three shots on Day 0, Day 7, and Day 21, respectively. For each shot, three different doses, including low dose $(0.125 \mu \mathrm{g} /$ dose $)$, middle dose $(0.5 \mu \mathrm{g} /$ dose $)$, and high dose $(2.0 \mu \mathrm{g} / \mathrm{dose})$, respectively, were used. Another 8 mice were injected with only adjuvant on Day 0, Day 7, and Day 21, serving as the control group. In order to compare the immunogenicity of the mutI-tri-RBD with the homo-tri-RBD, another two groups of BALB/c mice (6 mice in each group) were also immunized with the homo-tri-RBD plus aluminum hydroxide adjuvant by two shots of high dose $(2.0 \mu \mathrm{g} /$ dose $)$ or three shots of middle dose $(0.5 \mu \mathrm{g} /$ dose $)$. For all these groups, the sera of the immunized mice were collected at 7 days and 14 days after the last immunization.

\section{Mouse serum IgG measurement}

The titer of the RBD-specific IgG in the serum of the immunized mice was measured with ELISA by using monomeric His-tagged RBD of the SARS-CoV-2 prototype strain. His-tagged RBD was diluted with coating buffer to the concentration of $1 \mu \mathrm{g} / \mathrm{mL}$, which was coated on the 96-well plate with $100 \mu \mathrm{L}$ per well at $2-8{ }^{\circ} \mathrm{C}$ overnight. After washing 3 times with PBST, the plate was blocked using blocking buffer with $100 \mu \mathrm{L}$ per well and incubated at $37^{\circ} \mathrm{C}$ for $2 \mathrm{~h}$. Then, the plate was washed 3 times with PBST, and the serum samples were diluted by 2 -fold serial dilutions and added into the well with $100 \mu \mathrm{L}$ per well. After incubating the plate at $37^{\circ} \mathrm{C}$ for $1 \mathrm{~h}$ and washing 3 times with PBST, the HRPconjugated goat anti-mouse IgG at 1:10,000 dilution was added into the wells of the plate. The plate was again incubated at $37^{\circ} \mathrm{C}$ for $1 \mathrm{~h}$ and washed 3 times with PBST. Subsequently, a color reaction was performed for $10 \mathrm{~min}$ by adding the color development solution, which was then stopped using a sulfuric acidic solution. Both absorbances at 450 and $630 \mathrm{~nm}$ were measured and the difference between them $\mathrm{OD}_{450 / 630 \mathrm{~nm}}$ was obtained. To evaluate the titer of RBD-specific antibodies, the $\mathrm{OD}_{450 / 630 \mathrm{~nm}}$ value of the well without adding serum sample was taken as the blank control, and 2.1 times of the control value was used as the cutoff for positive results. The titer of RBD-specific IgG was determined as the reciprocal of the maximum dilution of the serum, where the $\mathrm{OD}_{450 / 630 \mathrm{~nm}}$ value is equal to or greater than the cutoff value.

\section{Pseudo-virus neutralization assay}

A total of 23 SARS-CoV-2 pseudo-viruses were used to evaluate the neutralizing antibody titers in the sera from immunized mice. The SARS-CoV-2 pseudotyped viruses used in the studies were constructed by using a vesicular stomatitis virus (VSV)-base system ${ }^{30-33}$. To evaluate the neutralization activities of the sera against these pseudoviruses, the serum sample was serially diluted fivefold at the starting dilution of 1:40 with the cell culture medium (DMEM containing 10\% Fetal Bovine Serum, $25 \mathrm{mM}$ HEPES and $1 \%$ penicillin-streptomycin). The serially diluted serum samples were added to the well of the plate with $50 \mu \mathrm{L}$ per well. The SARS-CoV-2 pseudo-virus was diluted 9 times with the cell culture medium to the titer of $1.3 \times 10^{4} \mathrm{TCID}_{50}$ per $\mathrm{mL}$ and added into the well using $50 \mu \mathrm{L}$ per well to mix with the serum, which was then incubated at $37^{\circ} \mathrm{C}$ for $1 \mathrm{~h} .50 \mu \mathrm{L}$ per well medium was also mixed with the pseudo-virus as control. $100 \mu \mathrm{L}$ per well medium served as blank. The HuH-7 cells were digested and suspended in the culture medium with a viable cell density of $2 \times 10^{5}$ per $\mathrm{mL}$, which were then added into the well with $100 \mu \mathrm{L}$ per well. After culture with $5 \% \mathrm{CO}_{2}$ at $37^{\circ} \mathrm{C}$ for $20-24 \mathrm{~h}$, the culture medium was removed, the cells were lysed, and the luciferase activity was evaluated as the relative light unit value. The neutralizing antibody titer was determined as the reciprocal of the dilution of the serum for $50 \%$ neutralization of viral infection, which was calculated using the Reed-Muench method.

\section{Live SARS-CoV-2 virus neutralization assay}

To evaluate the neutralization activity against the live SARS-CoV-2 virus, the live virus neutralization assay was performed in a BSL-3 laboratory of Guangdong Provincial Center for Disease Control and Prevention. Both the prototype (2020XN4276 strain), Delta (2020KXG0186 strain), and Beta (20SF18530 strain) viruses were used in the live virus neutralization assays. The mouse serum was serially diluted and mixed with an equal volume of $100 \mathrm{TCID}_{50}$ live SARS-CoV-2 virus. After incubation at $37^{\circ} \mathrm{C}$ for $2 \mathrm{~h}$, the serum-and-virus mixed solution was added into the well of the plate containing Vero-E6 cells with a density of $2 \times 10^{5}$ per $\mathrm{mL}$, which was then cultured at $37^{\circ} \mathrm{C}$ for $5-7$ days. Both cell and virus controls were also set up as a comparison. Subsequently, the inhibition of virus infection to the cells was observed, and the neutralizing antibody titer against the live SARS-CoV-2 virus was measured as the reciprocal of the serum dilution for $50 \%$ neutralization of viral infection.

\section{Live SARS-CoV-2 challenge experiment}

The mouse challenge experiments were performed in the BSL3 facility of the National Institute for Viral Disease Control and Prevention, Chinese Center for Disease Control and Prevention (China CDC), Beijing, China. A total of 40 female hACE2 B6-Tg transgenic mice (purchased from Beijing Huafukang Bioscience Co., Ltd, 
China.), aged 8-10 weeks and weighted $16-25 \mathrm{~g}$, were used for the live SARS-CoV-2 challenge experiment, which were randomly divided into eight groups (5 mice per group): three vaccine groups, three saline groups and two control groups. The mice in vaccine groups were immunized intramuscularly with two doses ( $2 \mu \mathrm{g} /$ dose $)$ of mutI-tri-RBD vaccine candidate on Day 0 and Day 21. In the saline and control groups, the mice were intramuscularly injected with physiological saline on Day 0 and Day 21. On day 7 after the second vaccination, the blood from the caudal vein of the immunized mice was collected, and serum was separated to measure the titer of neutralizing antibodies using a pseudo-virus neutralization assay. On day 14 after the second immunization, two vaccine groups, as well as corresponding two saline groups, were challenged with the prototype and Beta live SARS-CoV-2 viruses, respectively, and a control group was challenged with saline as a blank control. On day 19 after the whole immunization, the remaining one vaccine group and one saline group were challenged with the Delta live virus, and correspondingly a control group was challenged with saline as a blank control. In the challenge experiments, each animal was received $1.5 \times 10^{5} \mathrm{TCID}_{50}$ of live virus or $15 \mu \mathrm{L}$ saline via the intranasal route. During the challenge experiment, the changes in the body weight of the mice were measured, and the living status of the mice, including survival or not, muscle strength, vitality, and so on, were observed and recorded. Five days after the prototype and Beta virus challenges, or six days after

the Delta virus challenge, all mice were euthanized and the lung tissues were collected. The viral RNA was extracted from lung tissues by using a nucleic acid extraction kit (QIAGEN, No. 52906), and the viral load was evaluated by using a 2019-nCoV (N, ORF1ab, and S genes) nucleic acid detection kit (PCR-fluorescence probing). In addition, the histopathological sections of the lung tissues of the mice were stained with hematoxylin and eosin (H\&E), and a pathological examination was performed.

\section{Quantification and statistical analysis}

One-way analysis of variance with Sidak's multiple comparison test was used to determine the statistical significance of the difference between multiple groups, and Student's $t$-test was used for statistical analysis between two groups. ${ }^{*} P<0.05,{ }^{* * *} P<0.01$, ${ }^{* * * *} P<0.001$, ${ }^{* * * *} P<0.0001$, ns, not significant. Details can be found in the figure legend.

\section{Acknowledgements}

We thank Dr. Zhi Hua Xiao for assisting with protein expression. We also thank Professor Hui Wang and Dr. Qi Zhang for their assistance with biochemical tests and protein physico-chemical characterization. This work was supported by National Vaccine and Serum Institute (KTZC1900026C).

\section{Author details}

${ }^{1}$ The Sixth Laboratory, National Vaccine and Serum Institute (NVSI), Beijing, China. ${ }^{2}$ National Engineering Center for New Vaccine Research, Beijing, China. ${ }^{3}$ Guangdong Provincial Institute of Public Health, Guangdong Provincial Center for Disease Control and Prevention, Guangzhou, Guangdong, China. ${ }^{4}$ National Institute for Food and Drug Control (NIFDC), Beijing, China. ${ }^{5}$ National Institute for Viral Disease Control and Prevention, Chinese Center for Disease Control and Prevention (China CDC), Beijing, China. ${ }^{6}$ Qingdao Centers for Disease Control and Prevention, Qingdao, Shandong, China

\section{Author contributions}

Q.M.L., J.Z., and Y.L. conceived, designed, and supervised the experiments. J.G.S. conducted structure modeling and simulations. H.Z., F.J.S., Y.Q.J., J.W.H., Z.M.L., Y.N.H., X.F.Z., L.F.D., and S.S. performed protein expression, purification, and characterization. Z.Y.H. and P.H. verified the experimental methods. L.F.D., F.T., Z.J.M., X.F.Z., J.J.W., S.Y.R., W.J.H., M.Y.W., Z.B.H., Z.H.L., S.C., F.Z., and S.L.

performed mouse immunization, ELISA, and pseudo-virus neutralization assay. C.W.K. and R.Y.Y. conducted live-virus neutralization tests. G.Z.W., Y.L., X.Z., N.L., W.M.Z., K.X., R.Q.G., and W.Z. performed the mouse challenge experiments. Q.M. L., J.Z., J.G.S., Z.B.H., and Y.L. wrote and revised the manuscript.

\section{Data availability}

All data supporting the findings of this study are available within the paper or the Supplemental Information. Any additional information required to reanalyze the data reported in this paper is available from the corresponding author Q. M. L. (liqiming189@163.com) upon request.

\section{Conflict of interest}

Q.M.L., Y.L., J.Z., J.G.S., Y.Q.J., J.W.H., L.F.D., Z.B.H., X.F.Z., S.S., H.Z., F.T., Z.M.L., Y.N.H. S.C., Z.H.L., Z.J.M., F.Z., and N.L. are listed as inventors of the pending patent applications for the homo-tri-RBD and mutl-tri-RBD vaccines (application numbers: 202110348881.6, 202110464788.1, and 202110676901.2). The other authors declare no competing interests.

\section{Publisher's note}

Springer Nature remains neutral with regard to jurisdictional claims in published maps and institutional affiliations.

Supplementary information The online version contains supplementary material available at https://doi.org/10.1038/s41421-022-00383-5.

Received: 28 October 2021 Accepted: 30 January 2022

Published online: 15 February 2022

\section{References}

1. Zhou, P. et al. A pneumonia outbreak associated with a new coronavirus of probable bat origin. Nature 579, 270-273 (2020).

2. Hu, B., Guo, H., Zhou, P. \& Shi, Z. L. Characteristics of SARS-CoV-2 and COVID19. Nat. Rev. Microbiol. 19, 141-154 (2021).

3. Krammer, F. SARS-CoV-2 vaccines in development. Nature 586, 516-527 (2020).

4. Chakraborty, S., Mallajosyula, V., Tato, C. M., Tan, G. S. \& Wang, T. T. SARS-CoV-2 vaccines in advanced clinical trials: where do we stand? Adv. Drug Deliv. Rev. 172, 314-338 (2021).

5. Kyriakidis, N. C., López-Cortés, A., González, E. V., Grimaldos, A. B. \& Prado, E. O. SARS-CoV-2 vaccines strategies: a comprehensive review of pahse 3 candidates. Npj Vaccines 6, 28 (2021).

6. Krause, P. R. et al. SARS-CoV-2 variants and vaccines. N. Engl. J. Med. 385, 179-186 (2021).

7. Campbell, F. et al. Increased transmissibility and global spread of SARS-CoV-2 variants of concern as at June 2021. Eur. Surveill. 26, 2100509 (2021).

8. Choi, J. Y. \& Smith, D. M. SARS-CoV-2 variants of concern. Yonsei Med. J. 62 961-968 (2021).

9. Boehm, E. et al. Novel SARS-CoV-2 variants: the pandemics within the pandemic. Clin. Microbiol. Infect. 27, 1109-1117 (2021).

10. Zhou, D. et al. Evidence of escape of SARS-CoV-2 variant B.1.351 from natural and vaccine-induced sera. Cell 184, 2348-2361 (2021). 
11. Madhi, S. A. et al. Efficacy of the ChAdOx1 nCoV-19 Covid-19 vaccine against the B.1.351 variant. N. Engl. J. Med. 384, 1885-1898 (2021).

12. Edara, V. V. et al. Infection and vaccine-induced neutralizing antibody responses to the SARS-CoV-2 B.1.617.1 variant. N. Engl. J. Med. 385, 664-666 (2021).

13. Planas, D. et al. Reduced sensitivity of SARS-CoV-2 variant Delta to antibody neutralization. Nature 596, 276-280 (2021).

14. Zahradník, J., Nunvar, J. \& Schreiber, G. SARS-CoV-2 convergent evolution as a guide to explore adaptive advantage. bioRxiv https://doi.org/10.1101/ 2021.05.24.445534 (2021).

15. Martinez, D. R. et al. Chimeric spike mRNA vaccines protect against Sarbecovirus challenge in mice. Science 373, $991-998$ (2021).

16. Wrapp, D. et al. Cryo-EM structure of the 2019-nCoV spike in the prefusion conformation. Science 367, 1260-1263 (2020).

17. Sternberg, A.\& Naujokat, C. Stuctural features of coronavirus SARS-CoV-2 spike protein: Targets for vaccination. Life Sci. 257, 118056 (2020).

18. Piccoli, L. et al. Mapping Neutralizing and immunodominant sites on the SARS-CoV-2 spike receptor-binding domain by structure-guided high-resolution serology. Cell 183, 1024.e21-1042.e21 (2020).

19. Greaney, A. J. et al. Mapping mutations to the SARS-CoV-2 RBD that escape binding by different classes of antibodies. Nat. Commun. 12, 4196 (2021).

20. Greaney, A. J. et al. Comprehensive mapping of mutations in the SARS-CoV-2 receptor-binding domain that affect recognition by polyclonal human plasma antibodies. Cell Host Microbe 29, 463-476 (2021).

21. Yang, J. et al. A vaccine targeting the RBD of the $S$ protein of SARS-CoV-2 induces protective immunity. Nature 586, 572-577 (2020)

22. Liu, Z. et al. RBD-Fc-based COVID-19 vaccine candidate induces highly potent SARS-CoV-2 neutralizing antibody response. Sig. Transduct. Target. Ther. 5, 282 (2020).

23. Dai, L. et al. A universal design of betacoronavirus vaccines against COVID-19, MERS, and SARS. Cell 182, 722-733 (2020).

24. Dai, L. \& Gao, G. F. Viral targets for vaccines against COVID-19. Nat. Rev. Immun. 21, 73-82 (2021).

25. Webb, B. \& Sali, A. Comparative protein structure modeling using MODELLER. Curr. Protoc. Bioinform. 54, 5.6.1-5.6.37 (2016).

26. Abraham, M. J. et al. GROMACS: High performance molecular simulations through multi-level parallelism from laptops to supercomputers. SoftwareX 1-2, 19-25 (2015).

27. Touw, W. G. et al. A series of PDB related databases for everyday needs. Nucleic Acids Res. 43, D364-D368 (2015).

28. Kabsch, W. \& Sander, C. Dictionary of protein secondary structure: pattern recognition of hydrogen-bonded and geometrical features. Biopolymers 22, 2577-2637 (1983).

29. Lan, J. et al. Structure of the SARS-CoV-2 spike receptor-binding domain bound to the ACE2 receptor. Nature 581, 215-220 (2020).

30. Li, Q. et al. SARS-CoV-2 501Y.V2 variants lack higher infectivity but do have immune escape. Cell 184, 2362-2371 (2021).

31. Zhang, L. et al. Ten emerging SARS-CoV-2 spike variants exhibit variable infectivity, animal tropism, and antibody neutralization. Commun. Biol. 4, 1196 (2021).

32. Nie, J. et al. Establishment and validation of a pseudovirus neutralization assay for SARS-CoV-2. Emerg. Microbes Infect. 9, 680-686 (2020).
33. Nie, J. et al. Quantification of SARS-CoV-2 neutralizing antibody by a pseudotyped virus-based assay. Nat. Protoc. 15, 3699-3715 (2020).

34. Bergwerk, M. et al. Covid-19 breakthrough infections in vaccinated health care workers. N. Engl. J. Med. 385, 1474-1484 (2021).

35. Khoury, D. S. et al. Neutralizing antibody levels are highly predictive of immune protection from symptomatic SARS-CoV-2 infection. Nat. Med. 27, 1205-1211 (2021)

36. Earle, K. A. et al. Evidence for antibody as a protective correlate for COVID-19 vaccines. Vaccine 39, 4423-4428 (2021).

37. Barbeau, D. J. et al. Comparative analysis of human immune responses following SARS-CoV-2 vaccination with BNT162b2, mRNA-1273, or Ad26.COV2.S medRxiv https://doi.org/10.1101/2021.09.21.21262927 (2021).

38. Hassine, I. H. Covid-19 vaccines and variants of concern: a review. Rev. Med. Virol. https://doi.org/10.1002/rmv.2313 (2021).

39. Kleanthous, $H$. et al. Scientific rationale for developing potent RBD-based vaccines targeting COVID-19. Npj Vaccines 6, 128 (2021).

40. Yang, S. et al. Safety and immunogenicity of a recombinant tandem-repeat dimeric RBD-based protein subunit vaccine (ZF2001) against COVID-19 in adults: two randomised, double-blind, placebo-controlled, phase 1 and 2 trials. Lancet Infect. Dis. 21, 1107-1119 (2021).

41. SK bioscience. SK bioscience's COVID-19 vaccine will enter phase III clinical trial with promising interim data. https:/www.skbioscience.co.kr/en/news/ news_01_01?mode=view\&id=91\& (2021).

42. Zhao, X. et al. Neutralisation of ZF2001-elicited antisera to SARS-CoV-2 variants. Lancet Microbe 2, e494 (2021).

43. Huang, B. et al. Serum sample neutralization of BBIBP-CorV and ZF2001 vaccines to SARS-CoV-2 501Y.V2. Lancet Microbe 2, e285 (2021).

44. Logue, J. et al. Immunogenicity and in vivo protection of a variant nanoparticle vaccine that confers broad protection against emerging SARS-CoV-2 variants. bioRxiv https://doi.org/10.1101/2021.06.08.447631 (2021).

45. Su, D. et al. Broad neutralization against SARS-CoV-2 variants induced by a modified B.1.351 protein-based COVID-19 vaccine candidate. bioRxiv https:// doi.org/10.1101/2021.05.16.444369 (2021).

46. Wu, K. et al. Variant SARS-CoV-2 mRNA vaccines confer broad neutralization as primary or booster series in mice. Vaccine 39, 7394-7400 (2021).

47. Cohen, A. A. et al. Mosaic nanoparticles elicit cross-reactive immune responses to zoonotic coronaviruses in mice. Science $\mathbf{3 7 1}, 735-741$ (2021).

48. Walls, A. C. et al. Elicitation of broadly protective sarbecovirus immunity by receptor-binding domain nanoparticle vaccines. Cell 184, 5432-5447 (2021).

49. Shen, M. Y. \& Sali, A. Statistical potential for assessment and prediction of protein structures. Protein Sci. 15, 2507-2524 (2006).

50. Pettersen, E. F. et al. UCSF Chimera - a visualization system for exploratory research and analysis. J. Comput. Chem. 25, 1605-1612 (2004).

51. Mané, C. et al. Assessment of the molecular weight distribution of tannin fractions through MALDI-TOF MS analysis of protein-tannin complexes. Anal. Chem. 79, 2239-2248 (2007)

52. Jang, Y. R. et al. Development of an optimized MALDI-TOF-MS method for high-throughput identification of high-molecular-weight glutenin subunits in wheat. Molecules 25, 4347 (2020)

53. Micsonai, A. et al. BeStSel: a web server for accurate protein secondary structure prediction and fold recognition from the circular dichroism spectra. Nucleic Acids Res. 46, W315-W322 (2018). 